\title{
USO DE LA CATEGORÍA "TRASTORNO ANTISOCIAL DE LA PERSONALIDAD" COMO INVISIBILIZACIÓN DE LA VIOLENCIA FEMINICIDA
}

\footnotetext{
${ }^{1}$ Secretaría General de Gobierno del Estado de México, Ecatepec de Morelos, México.ramoswaldo@yahoo.com.mx
}

\author{
JESÚS OSWALDO \\ HERRERA RAMOS ${ }^{1}$
}

\section{Resumen}

El presente ensayo tiene como objetivo examinar, desde una perspectiva feminista, las conductas antisociales de los hombres con Trastorno Antisocial de la Personalidad (TAP) como actos de violencia feminicida. Desde esta perspectiva, el psicópata, criminal o no, constituye la expresión máxima de la violencia feminicida: es el extremo negativo de la dimensión de la masculinidad hegemónica. Para Russell (2006), estar mentalmente enfermo no libera a los hombres de su misoginia o su racismo; hay una falta de consideración de los factores socioculturales que contribuyen a las causas de los actos criminales. Los actos y las conductas violentas contra las mujeres, entonces, se deben visibilizar como una expresión misógina que atenta contra los derechos humanos de las mujeres (Lagarde, 2008). No es viable 
la solución del problema del trastorno antisocial sólo por la perspectiva de la psiquiatría, pero sí lo es que el problema de violencia feminicida misógina pueda y deba ser erradicado a través de modificaciones en las estructuras legales y sociales, propuestas por los movimientos feministas. ${ }^{2}$

Palabras clave: violencia feminicida, trastorno antisocial de la personalidad, psicopatía, misoginia y violencia de género.

\section{Abstract}

The present essay aims to examine, from a feminist perspective, the antisocial behavior of men who suffer from Antisocial Personality Disorder, like acts of femicide violence. From this perspective, the psychopath, criminal or not, constitutes the maximum expression of femicide violence: It is the negative extreme of the hegemonic masculinity dimension. For Russell (2006), being mentally ill does not exempt men from their misogyny and racism; there is a lack of consideration of the sociocultural factors which promote the causes of the criminal acts. The acts and the violent behaviors against women, then, have to be displayed as a misogynist expression which violates the human rights of all women (Lagarde, 2008). A solution to this problem using only a psychiatric perspective is not viable. A solution is that the femicide violence problem and misogynist can and should be 
eradicated through the modifications in the legal and social structures, proposed in the feminist movements.

Keywords: feminicide violence, antisocial personality disorder, psychopathy, misogyny and gender violence.

RECEPCIÓN: 05 DE MARZO DE 2013/ ACEPTACIÓN: 13 DE AGOSTO DE 2015

\section{LOS TRASTORNOS MENTALES,} ¿UNA CATEGORÍA O UNA DIMENSIÓN?

Es tema de debate en la psicología el planteamiento siguiente: ilos trastornos de la personalidad existen a lo largo de dimensiones que reflejan variantes extremas de la personalidad "normal", o son categorías expresas cualitativamente diferentes y claramente desmarcadas de los rasgos de la personalidad "normal" y entre sí? (Phillips y Gunderson, 1996).

${ }^{3}$ El DSM-IV-TR considera equivalentes el trastorno antisocial de la personalidad y la psicopatía, por lo que se usaran indistintamente tales términos. En adelante, se usará TAP en lugar de trastorno antisocial.
Dentro de la personalidad psicopática o trastorno antisocial de la personalidad ${ }^{3}$ hablaremos de una dimensión continua; es decir, algunos hombres presentan rasgos psicopáticos o antisociales más psicopatológicos que otros. Amor, Echeburúa y Loinaz (2009) se preguntan si existe el maltratador antisocial o se trata más bien de un continuo antisocial, es decir, se puede 


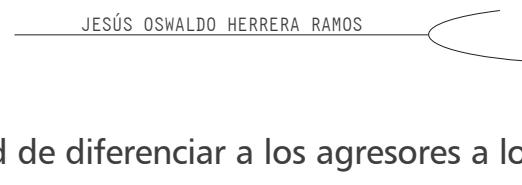

plantear la posibilidad de diferenciar a los agresores a lo largo de un continuo antisocial. Para estos autores, clasificar en tipologías a los hombres maltratadores sólo es con el objetivo de determinar las necesidades y alcances terapéuticos de cada uno de los agresores.

La violencia es una característica presente en muchos de los trastornos mentales que abarca el Manual Diagnóstico y Estadístico de los Trastornos Mentales (American Psychiatric Association, 2002); ${ }^{4}$ por ejemplo, trastornos esquizofrénicos, trastornos relaciona${ }^{4}$ En adelante se citará como el DSM-IV-TR o el DSM-V dos con sustancias, trastornos de la personalidad, trastorno explosivo intermitente, episodios maníacos.

Entre estos últimos, el TAP sobresale, entre sus criterios diagnósticos, por un patrón general de desprecio y violación de los derechos de los demás, que se acompaña, entre otras características, por impulsividad, irritabilidad y agresividad, despreocupación imprudente por la seguridad de los demás, falta de remordimientos, etc.

Según Spitzer, Gibbon, Skodol, Williams y First (2003), una de las críticas hacia los criterios para el TAP, en el DSM-IV-TR, es el excesivo énfasis que se da a los actos antisociales, que pueden ocasionar problemas legales, así como la poca atención en los rasgos psicológicos del trastorno, como ausencia de culpa, falta de lealtad hacia las personas y carencia de empatía. La 
crítica del presente trabajo apuntaría hacia una subrepresentación de los hombres en esta categoría psicopatológica, en el sentido de que una gran variedad de actos antisociales de éstos no conllevan necesariamente a problemas legales en las sociedades patriarcales, por tal razón, muchos de ellos podrían evitar las consecuencias legales de sus conductas violentas, y por consiguiente no caer en la categoría de TAP.

EI DSM-IV-TR refiere que la prevalencia del TAP, en las muestras de población general, es de aproximadamente $3 \%$ en hombres y $1 \%$ en mujeres. De acuerdo con la Encuesta Nacional de Epidemiología Psiquiátrica (Medina-Mora et al., 2003), en México, un $10.3 \%$ de hombres han padecido trastorno disocial (antecedente del TAP) alguna vez en su vida, mientras que sólo un $2.3 \%$ de las mujeres se han visto afectadas.

Sin embargo, habría que preguntarnos por qué autores especializados (Hare, 2003; Spitzer et al., 2003), al relatar casos del TAP, mencionan casi exclusivamente hombres, mientras las mujeres constituyen las principales o únicas víctimas de éstos. Incluso cuando el TAP afectara, como proporción, un máximo de tres veces más a los hombres que a las mujeres, esta estadística nos plantea cómo incide y afecta el género en dicho trastorno, y permite reflexionar si las personas con tal trastorno, más que conducirse con misantropía, actúan motivados por la misoginia. En las contadas excepciones que Hare (2003) describe mujeres psicópatas, éstas ejercen mucha menos violencia que los hombres, y a veces se encuentran en el límite entre lo legal y lo ilegal. 


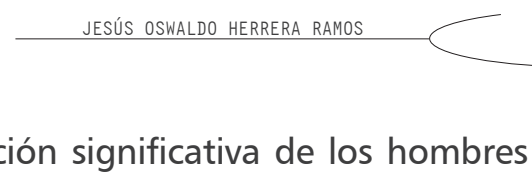

Según este autor, una proporción significativa de los hombres psicópatas son maltratadores habituales hacia sus parejas, lo cual permite asociar a la psicopatía como violencia de género.

No es casualidad que los psicópatas tengan entre sus víctimas, principal o exclusivamente, a mujeres. Los crímenes cometidos por psicópatas tienen implicaciones políticas hacia el género; es decir, son asesinatos basados en un sistema de supremacía masculina (Caputi, 1993). El psicópata, criminal o no, constituye la expresión máxima de la violencia feminicida. La Ley General de Acceso de las Mujeres a una Vida Libre de Violencia define la violencia feminicida como:

La forma extrema de violencia de género contra las mujeres, producto de la violación de sus derechos humanos, en los ámbitos público y privado, conformada por el conjunto de conductas misóginas que pueden conllevar impunidad social y del Estado y puede culminar en homicidio y otras formas de muerte violenta de mujeres (2011:6).

\section{LA ENFERMEDAD MENTAL COMO METÁFORA DE LA ENFERMEDAD FÍSICA}

Los editores del DSM-IV-TR afirman que no existe una definición que especifique adecuadamente los límites del concepto "trastorno mental", es decir, carece de una 
definición operacional; concordando en que los trastornos mentales se definen a partir de diferentes niveles de abstracción. Estos editores aseveran que "el término 'trastorno mental' implica, desafortunadamente, una distinción entre trastornos mentales y físicos (un anacronismo reduccionista del dualismo mente/cuerpo); los conocimientos actuales indican que hay mucho de 'físico' en los trastornos mentales".

Sin embargo, para una gran parte de los trastornos mentales, especialmente el TAP, no explican cuál es la parte física, hormonal u cerebral del trastorno en cuestión. Muchos psiquiatras creen que las anormalidades cerebrales (por ejemplo, surcos cerebrales anormalmente aumentados y ventrículos ligeramente dilatados) pueden ser un factor etiológico en el desarrollo de su conducta patológica. Sin embargo, Spitzer et al. (2003) se preguntan si tales alteraciones son más bien el resultado de frecuentes traumatismos craneales. Esto considerando que los mismos psicópatas fueron víctimas de severos abusos físicos en la infancia, y que, además, siendo frecuentes las peleas físicas con otras personas, y sin preocuparles su propia seguridad, suelen ser blancos fáciles de accidentes que terminen en traumatismos.

Detrás de todo esto, está el cuestionamiento más general sobre las enfermedades mentales. Thomas Szasz (2002), controvertido psiquiatra y pionero del movimiento de la antipsiquiatría, afirma que el concepto de la enfermedad mental ha sido abstracto y teórico, más que empírico y concreto; dicho 


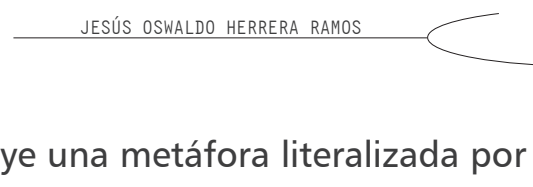

concepto psiquiátrico constituye una metáfora literalizada por razones prácticas, convirtiéndose la psiquiatría en el estudio "científico" del mal comportamiento y su control "médico". El diagnóstico de las enfermedades mentales, para la psiquiatría, se fundamenta en los "síntomas del comportamiento" de los supuestos pacientes. Sin embargo, para que una condición sea nombrada enfermedad mental debe cumplir con los siguientes requerimientos:

1) Decir que una condición es una enfermedad mental equivale a decir que la persona no es responsable por su padecimiento y, por lo tanto, no aplican sanciones, por lo menos de tipo legal. Ésta es una característica de la enfermedad en general. La aparición de la enfermedad no puede ser explicada como el resultado de una decisión voluntaria, independientemente de las ganancias secundarias que ésta pueda reportar.

2) Considerar algo una enfermedad mental equivale a decir que es "un tipo de cosa" susceptible de ser tratada y, en el mejor de los casos, curada por medio de una terapéutica médica (Morgan, 1975 en Ordorika, 2005).

\section{EL CUESTIONAMIENTO \\ DEL USO DE LA ETIQUETA TAP COMO CATEGORÍA MENTAL}

El psicópata no está trastornado, es estable; constituye una manera de ser, de estar y de relacionarse con el mundo; reducir la psicopatía a un trastorno de 
personalidad implica un grave error de comprensión clínica de psicopatología (Jáuregui, 2008). La psicopatía o TAP no se circunscribe exclusivamente a la conducta criminal, sino que también está presente en personas aparentemente normales, adaptadas e integradas en la sociedad y en el mundo laboral (Jáuregui, 2008; Millon et al., 2004). Además, la literatura psicológica señala que actualmente no hay ninguna terapia psicológica eficaz para el TAP; los escasos tratamientos psicológicos propuestos son dudosos en cuanto a su eficacia clínica (Quiroga y Errasti, 2001).

La idea central de este ensayo dista de considerar la enfermedad mental como desvinculación de la responsabilidad por las consecuencias de la conducta. Todavía hoy es común la idea de que "el enfermo mental es víctima y sus procesos mentales son los victimarios; el delincuente es una víctima, una persona que ha tenido una vida familiar y social destructiva, carente de equilibrio y estabilidad emocional" (Hikal, 2009: 130).

\section{¿IMPUTABILIDAD}

\section{O INIMPUTABILIDAD DEL TAP?}

En países como España, para que la imputabilidad del sujeto se vea anulada o atenuada, su capacidad cognoscitiva y volitiva tendrá que verse afectada en el momento de comisión del delito y tener una relación causal con éste (Molina, Trabazo, López y Fernández, 2009). Hay autores que piensan que los psicópatas no poseen la misma capacidad de 


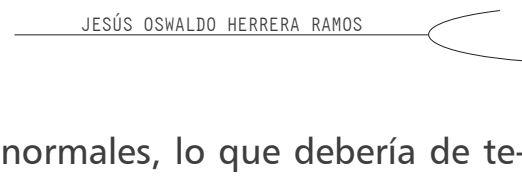

autocontrol que las personas normales, lo que debería de tenerse en cuenta al determinar su tratamiento jurídico, aunque fuese para atenuar la responsabilidad penal (Vega, 2004).

Otras autoras, como Barbero y Salduna (2007), afirman que no se puede asegurar que los psicópatas son siempre imputables o inimputables; es decir, no es suficiente el diagnóstico del TAP, sino que se procederá según las circunstancias específicas del caso concreto, y de la gravedad del trastorno. En nuestro contexto jurídico, el Código Penal Federal de nuestro país, en su capítulo IV, artículo 15, fracción VII, estipula como causa de exclusión del delito que:

Al momento de realizar el hecho típico, el agente no tenga la capacidad de comprender el carácter ilícito de aquél o de conducirse de acuerdo con esa comprensión, en virtud de padecer trastorno mental o desarrollo intelectual retardado, a no ser que el agente hubiere preordenado su trastorno mental dolosa o culposamente, en cuyo caso responderá por el resultado típico siempre y cuando lo haya previsto o le fuere previsible.

Herrero y Colom (2006) señalan, en su estudio, que los internos de una población penitenciaria exhiben menor impulsividad comparados con la población normal, cuestionando, de esta forma, el modelo de comportamiento antisocial basado en las dificultades temperamentales de los delincuentes. Esto es importante ya que si se considera que no se tiene control de los impulsos, entonces se cuestiona la capacidad mental, lo cual tiene implicaciones relevantes, ya que la incapacidad de 
obrar puede ser usado como mecanismo para evitar responsabilidades (Gómez-Jarabo, Rodríguez y Olavarrieta, 2004).

Las conductas de los psicópatas no son, entonces, resultado de unas mentes trastornadas. Según los cánones legales y psiquiátricos, son conscientes y responsables de las consecuencias de sus actos, aunque se muestren incapaces para tratar a los demás como seres humanos pensantes y sensibles (Hare, 2003). En sentido estrictamente jurídico-psicológico, estos sujetos tienen conocimiento de la ilicitud de sus acciones y la voluntad determinante al infringir la norma legal, por lo que son responsables legales de sus actos (Molina et al., 2009).

\section{¿CRIMINALIZARLOS}

\section{O PATOLOGIZARLOS, O AMBOS?}

Margarita Tirado-Álvarez enlista una serie de razones para aumentar las sanciones penales impuestas a los psicópatas criminales, planteando la posibilidad, incluso, de la aplicación perpetua de la pena privativa de libertad:

Primero, aunque el individuo pague una pena prolongada, nunca se resocializará dada su condición de incurable; segundo, el mismo no sentirá culpa por cuanto no la experimenta ni aprenderá de los errores cometidos; tercero, dado el alto nivel de reincidencia, una vez cumplida la pena el sujeto seguramente repetirá su conducta; cuarto, la retribución sólo cabría en la medida en que 
repare civilmente y satisfaga el deseo de la víctima o sus familiares de ver al victimario aislado de la sociedad a la que pueda dañar; quinto, la prevención especial no será posible porque no interioriza la norma, además, porque el alto nivel de reincidencia que los caracteriza, su incorregibilidad, la impulsividad y la ausencia de temor al castigo no evitarán que el sujeto vuelva a incurrir en la conducta que originó la sanción penal (2010: 129-130).

\section{LA PSIQUIATRÍA Y EL CONTROL}

\section{SOCIAL DE LAS MUJERES}

La psiquiatría como institución patriarcal tiene fuertes sesgos sexistas. Como ejemplo, pensemos en las sobrerrepresentación de las mujeres en las estadísticas epidemiológicas psiquiátricas en el DSM-IV-TR, especialmente en los trastornos del estado de ánimo, como la depresión. Más aún, las categorías de salud y padecimientos mentales tienen un carácter de construcción social e histórico (Ordorika, 2009).

La sobrerrepresentación de las mujeres en las estadísticas epidemiológicas psiquiátricas es consecuencia de que las condiciones de vida de las mujeres estén marcadas por la opresión y dominación de los hombres y el sistema patriarcal (Burín, 1999).

De la misma forma, se puede argumentar que la mayor prevalencia de hombres en los trastornos violentos, especialmente el TAP, es resultado del sistema patriarcal hegemónico 
que les concede, al colectivo de los hombres, el poder y control sobre las mujeres, el cual deriva en el ejercicio de la violencia de género. De cualquier forma, cabria preguntarse si incluir un trastorno que describa al hombre que ejerce violencia de género contra las mujeres reportaría más ventajas que inconvenientes. Castro y Bronfman (1993: 386) se preguntan: “ipor qué la mayor predisposición a la violencia de parte de los hombres no es clasificada como enfermedad mental?".

Irma Saucedo (2003), en contraparte, afirma que las instituciones de salud, al clasificar los actos violentos y sus consecuencias como enfermedades o patologías individuales, validan las formas de poder y control que son ejercidas por los hombres contra las mujeres en la sociedad, es decir, patologizar a éstos desmarcaría el problema de la violencia contra las mujeres como una cuestión estructural y de género, y lo situaría solamente en un plano individual.

\section{GÉNERO, PODER Y PATRIARCADO}

El género es una categoría social asignada a un cuerpo sexuado; es también una manera de diferenciar los roles sociales asignados a mujeres y hombres. La relevancia del tema de género radica en que éste es un elemento establecido de las relaciones sociales; además es el terreno fundamental dentro del cual o por medio del cual se configura el poder (Scott, 1990). 
Para Foucault (1980), en cualquier sociedad hay múltiples relaciones de poder, las cuales permean, caracterizan y constituyen el cuerpo social, y estas relaciones no pueden por sí mismas ser establecidas o consolidadas sin el funcionamiento de un discurso. El poder está en todas partes, viene de todas partes, se ejerce a partir de innumerables puntos y en el juego de relaciones no igualitarias (Foucault, 1977).

Es aquí donde las autoras feministas hablan de cultura y discurso de género. Lamas (2007) sostiene que lo que sobresale persistentemente en la trama de género de la cultura es que la diferencia se traduce en desigualdad. Dicho proceso convierte al género en el fundamento de la subordinación social de las mujeres.

La cultura, como tal, cumple un papel relevante, tanto en la reproducción y perpetuación de patrones de dominación de los hombres sobre las mujeres, como en la modificación de las desiguales relaciones de género prevalentes en Latinoamérica (Castro y Cacique, 2008). Incluso, la cultura es el resultado de la manera en que la sociedad interpreta las diferencias entre mujeres y varones (Lamas, 2002). Es así que el género estructura el modo en que perciben los sujetos sociales y moldea la forma en que se organiza la vida social.

Esto significa que la socialización tiene una clara influencia tanto en el desarrollo de la identidad de género, como en la aceptación de los roles de género, las conductas de rol, o en el desarrollo de una determinada ideología sobre la masculinidad 
o el feminismo; además, tales variables se relacionan con la discriminación de género (Castillo y Montes, 2007).

La marcada desigualdad, entre varones y mujeres, en las esferas económica, educativa, política y legal de la sociedad mantiene una estructura patriarcal o de desigualdad de género, que influye directa o indirectamente en las experiencias de discriminación y de violencia hacia las mujeres (Frías, 2008). Lo que ahora llamamos violencia, algún tiempo atrás se contemplaba como una legitima manifestación del poder y una forma consensuada de interactuar en las relaciones sociales (Perrone y Nannini, 2007).

La violencia de género alude a los mecanismos a través de las cuales se intenta mantener el sistema de jerarquías impuesto por el patriarcado; uno de los obstáculos principales para la comprensión de la violencia de género contra las mujeres ha sido estructurada a partir de dos ejes fundamentales: la invisibilización y la naturalización (Corsi, 2003).

Siendo así, debemos considerar necesariamente la ideología patriarcal al hablar de la violencia de los hombres contra las mujeres (White, 1994). Ya que la estructura del patriarcado, construido sobre el modelo de la dominación de los hombres sobre el cuerpo y la voluntad de las mujeres, es responsable del sexismo y de la violencia hacia las mujeres (Gargallo, 2006). Judith Butler cita que:

Esta violencia emerge de un profundo deseo de mantener el orden del género binario natural o necesario, 
de convertirlo en una estructura, ya sea natural, cultural o ambas, contra la cual ningún humano pueda oponerse y seguir siendo humano... entonces parece que la violencia emerge precisamente como una demanda de deshacer dicho reconocimiento, de cuestionar su posibilidad, de convertirlo en irreal e imposible frente a cualquier apariencia de lo contrario (2006: 59).

\section{MISOGINIA,}

\section{VIOLENCIA FEMINICIDA Y TAP}

En el DSM-IV-TR no hay ningún trastorno que haga referencia al hombre que ejerce violencia de género contra las mujeres. Teresa Ordorika ${ }^{5}$ (comunicación personal) entiende que la categoría de trastorno mental también exime de responsabilidad, y que no todo comportamiento inmoral o antisocial constituye un trastorno, ya que tales comportamientos están más relacionados con las condiciones estructurales del ejercicio del poder. Clasificar o no tales comportamientos como categorías psicopatológicas tiene relevantes implicaciones políticas.

Otra de las posibles razones se relaciona con la definición anterior de que los trastornos clasificados en ese manual deben apartarse de las expectativas culturales. Sin embargo, en las sociedades patriarcales, en distintos grados y modalidades, la violencia de género sigue siendo relativamente aceptada y 
tolerada como forma legítima de resolver los conflictos. Dentro de este marco de tolerancia, la violencia se dirige principalmente contra las mujeres debido a la relación de desigualdad que conserva frente a los hombres, producto de la socialización de género (Lagarde, 2008; Saldívar, Ramos y Saltijeral, 2004).

La misoginia, como pilar básico de la violencia de género, alude a una mezcla de temor, rechazo y odio contra las mujeres, a la estructura fundamental del dominio masculino, la impronta de las relaciones sociales y de las concepciones hegemónicas de la realidad social; la misoginia tiene también como manifestación la enajenación de los hombres (Cazés, 2005). La enajenación es el caldo de cultivo para la violencia.

Además, las conductas misóginas son grotescas y aberrantes, pero quienes las ejecutan son sujetos ordinarios caracterizados por una falta de conciencia social y emocional, no son monstruos o seres satánicos, carentes de una convicción ideológicamente mínimamente elaborada (Zumaya, 2005). Marcela Lagarde refiere que:

La violencia feminicida se produce por la organización social genérica patriarcal, jerárquica, de supremacía e inferioridad, que crea desigualdad de género entre mujeres y hombres. Se produce, también, por la aceptación y la tolerancia, que evidencian múltiples complicidades entre hombres supremacistas, machistas y misóginos, 


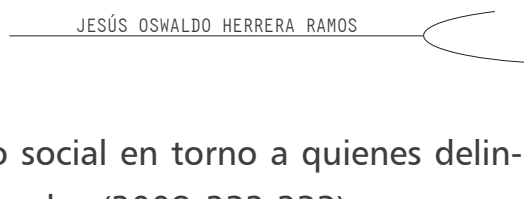

incluso por el silencio social en torno a quienes delinquen y no son sancionados (2008: 232-233).

No obstante, no todos los criminales son antisociales y no todos los antisociales son criminales; hay muchas características antisociales en el rango de lo normal que son alentadas y admiradas en nuestra sociedad competitiva (Millon et al., 2004). Una de las razones principales de que las mujeres sean el blanco principal de los psicópatas la podemos encontrar en la vulnerabilidad o falta de sus derechos humanos, producto de la misoginia. Marcela Lagarde afirma que "las mujeres no son sujetas de derecho ni son consideradas ni tratadas como ciudadanas" (2008: 234).

\section{PERFIL DEL PSICÓPATA Y PERFIL DEL HOMBRE MALTRATADOR CONTRA SU PAREJA}

El DSM-V, la nueva edición del Manual Estadístico y Diagnóstico de los Trastornos Mentales, refiere que las personas con TAP desprecian los deseos, derechos o sentimientos de los demás; frecuentemente, engañan y manipulan con tal de conseguir provecho o placer personales (por ejemplo, para obtener dinero, sexo o poder). Los sujetos tienden a ser irritables y agresivos y pueden tener peleas físicas repetidas o cometer actos de agresión (incluidos los malos tratos al cónyuge). Tienen pocos o nulos remordimientos por 
las consecuencias de sus actos; pueden ser indiferentes o dar justificaciones superficiales por haber ofendido, maltratado o robado a alguien. Estas personas pueden culpar a las víctimas por ser tontos, débiles o por merecer su mala suerte, pueden minimizar las consecuencias desagradables de sus actos o, simplemente, mostrar una completa indiferencia.

El perfil del psicópata no es tan diferente del perfil del hombre violento. Echeburúa y Fernández-Montalvo (1998) enlistan las siguientes características del perfil del hombre violento: se irrita fácilmente cuando se le ponen límites, no controla sus impulsos, comete actos de violencia cuando se enoja, ha maltratado a otras mujeres, culpa a otros de sus problemas, excesivamente celoso y posesivo, experimenta cambios bruscos de humor, bebe alcohol en exceso, tiene una baja autoestima, y cree que las mujeres deben estar subordinadas a los hombres. Aun cuando todos estos rasgos pueden estar presentes en los psicópatas, por lo menos las cinco principales características coinciden con criterios diagnósticos del TAP.

EI DSM-IV-TR asevera que el maltrato o el abandono en la infancia, el comportamiento inestable o variable de los padres o la inconsistencia en la disciplina por parte de los padres aumentan las probabilidades de que un trastorno disocial evolucione hasta un TAP. Es decir, el estudio del maltrato infantil y el abuso sexual en la infancia aportaría antecedentes psicógenos asociados a la aparición de conductas antisociales (Alarcón, Vinet y Salvo, 2005). 
No obstante, Echeburúa y Fernández-Montalvo (1998) aseguran que el $36 \%$ de los maltratadores han sido víctimas de malos tratos en la niñez, mientras el $64 \%$ no ha sufrido maltrato en la infancia. Estas cifras debilitan el argumento que sostiene que el factor determinante de la violencia de los hombres contra las mujeres radica en el hecho de ellos mismos haber sido violentados en su infancia, con ello se valida la tesis de que la violencia de género está vinculada con las condiciones estructurales del ejercicio del poder en el patriarcado.

Además, el DSM-V presenta a los sujetos con TAP como frecuentemente carentes de empatía, con tendencia a ser insensibles, cínicos y a menospreciar los sentimientos, derechos y penalidades de los demás. También pueden ser irresponsables y explotadores en sus relaciones sexuales; tener una historia de muchos acompañantes sexuales y carecer de una relación monógama duradera, además de poseer una tendencia a la irresponsabilidad paterna. Aunque las estadísticas refieran que sólo un pequeño porcentaje de los hombres que ejercen violencia contra sus parejas se pueden clasificar en un trastorno de personalidad, la mayoría de ellos o todos, en algunos aspectos de su conducta, revelan un rasgo o característica típica del TAP, los cuales son, como ya se ha mencionado antes, impulsividad, irritabilidad y agresividad, despreocupación imprudente por la seguridad de los demás, falta de remordimientos, entre otros.

"Con una total carencia de conciencia y sentimientos por los demás, toman lo que les apetece de la forma que les viene en 
gana, sin respeto por las normas sociales y sin el menor rastro de arrepentimiento o piedad" (Hare, 2003: 1). Esta descripción sobre los psicópatas, así como los criterios diagnósticos del DSMIV-TR sobre el TAP, nos recuerdan mucho a los hombres que ejercen violencia de género contra las mujeres, pareciera que no hay diferencias cualitativas, sólo diferencias de grado. El psicópata es, entonces, el extremo negativo de la dimensión de la masculinidad hegemónica. Recordemos lo que Kaufman (1994) afirma que mucho de lo que las personas asocian con masculinidad gira sobre la capacidad del hombre de ejercer poder y control.

Usualmente, los psicópatas no experimentan el malestar subjetivo que acompaña a casi todos de los más de 300 trastornos mentales del DSM-IV-TR, característica que, también, se encuentra en la mayoría de los hombres que ejercen violencia feminicida.

\section{CONCLUSIONES}

La intención de este ensayo no es patologizar a los hombres que ejercen violencia de género contra las mujeres (lo cual no sirve de mucho ya que externalizamos el problema), o identificar a estos últimos con psicópatas. Beyebach (1999) sostiene que el uso de etiquetas diagnósticas como patológicas supone hablar de entidades individuales, aisladas de su contexto sociocultural.

Una de las premisas que subyace aquí es considerar las clasificaciones de la psicopatología como dimensiones continuas 


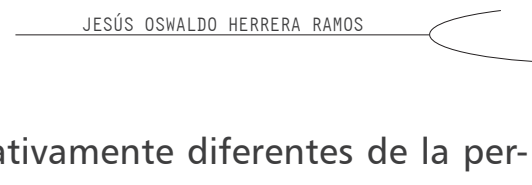

en lugar de categorías cualitativamente diferentes de la personalidad "normal". Considerar a los hombres psicópatas como trastornados mentales sólo facilita su exculpación, y menosprecia la influencia del orden desigual de género que acepta y tolera la violencia de género contra las mujeres, sin que por ello estos hombres dejen de ser totalmente responsables, legal y moralmente, por las consecuencias de sus actos violentos.

Es válido cuestionarse cual es la utilidad de relacionar conceptualmente la violencia feminicida con la psicopatía. La justificación es reconocer y visibilizar los actos psicopáticos como violencia feminicida y no verlos exclusivamente como manifestaciones de un trastorno mental, aislados de su contexto social. Teresa Ordorika sostiene que:

Las investigaciones feministas deben ser capaces de relacionar los padecimientos mentales tanto con los contextos inmediatos de las personas, como con las estructuras sociales en las que prevalecen desigualdades que afectan negativamente la salud, con la finalidad de ubicar qué tipo de acciones son necesarias para resolverlos (2009: 667).

No es casualidad que el trastorno violento por excelencia, el TAP, haya sido categorizado sólo como desorden mental. Podría decirse que no es viable el problema del trastorno antisocial 
para ser solucionado sólo por la psiquiatría, pero sí lo es que el problema de violencia feminicida misógina pueda y sea erradicado a través de modificaciones en las estructuras legales y sociales propuestas por los movimientos feministas.

Diana Russell (2006) afirma que estar mentalmente enfermo no libera a los hombres de su misoginia o su racismo; hay una falta de consideración de los factores socioculturales que contribuyen a las causas de los actos criminales. La misma autora enfatiza que los feminicidios y la violencia feminicida son trivializados y despolitizados con el argumento de que los hombres que ejercen dichos actos criminales están trastornados.

Los actos y conductas misóginas de violencia feminicida que ejercen los hombres psicópatas no se pueden reducir sólo a actos individuales o psicopatológicos, sin ocultar, al mismo tiempo, la relación de poder patriarcal que sustenta el orden de género. Por las anteriores razones, no es posible hablar de actos y conductas violentas feminicidas exclusivamente como psicopatologías individuales, sino principalmente como trastornos sociales del sistema patriarcal dominante, o como desórdenes estructurales de la sociedad hegemónica. Teresa Ordorika (2009) enfatiza que la salud mental está estrechamente vinculada con las condiciones de vida de las personas.

Tales actos y conductas violentas contra las mujeres se deben visibilizar como una expresión misógina que atenta contra los derechos humanos de las mujeres (Lagarde, 2008). Esta 


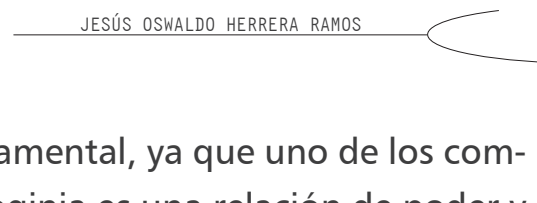

perspectiva de género es fundamental, ya que uno de los componentes relevantes de la misoginia es una relación de poder y control; todos los hombres, querámoslo o no en lo individual, somos estructuralmente misóginos (Minello, 2005).

Cuando se discute sobre la problemática de la violencia de los hombres contra las mujeres no se puede dejar de hacerlo desde una perspectiva de género. De no hacerlo así incurriríamos en un grave error: soslayar el contexto y reducir el problema a circunstancias de índole individual. Peor aún acabaríamos por culpabilizar a la víctima de su situación y colocando a ambos, víctima y victimario, en el mismo nivel de responsabilidad.

Resulta, entonces, que la violencia feminicida constituye el verdadero malestar en la cultura. Nos resulta difícil aceptar y tolerar que vivamos en una sociedad sexista y misógina, pero solamente este reconocimiento explícito posibilita otras muchas condiciones que, a su vez, permitan la transformación de las estructuras sociales, culturales, políticas y legales que sustentan el orden patriarcal.

\section{BIBLIOGRAFÍA}

AlARcón, P., Vinet, E. y SALVo, S. (2005). “Estilos de personalidad y desadaptación social durante la adolescencia", en Psykhe, vol. 14, t. 1, pp. 3-16.

AMERICAN PSYCHIATRIC ASSOCIATION (2002). Manual diagnóstico y estadístico de los trastornos mentales: texto revisado. Barcelona: Masson. 
AMOR, P.; ECHEBURÚA, E. y LOINAZ, I. (2009). "Se puede establecer una clasificación tipológica de los hombres violentos contra la pareja", en International Journal of Clinical and Health Psychology, vol. 9, núm. 3, pp. 519-539.

BARbero, N. y SAldunA, M. (2007). "Responsabilidad penal del psicópata", en Revista Latinoamericana de Derecho, vol. IV, pp. 89-127. Recuperado el 7 de junio del 2011 de: http://www.juridicas.unam.mx/publica/librev/ $\mathrm{rev} / \mathrm{revlad} / \mathrm{cont} / 7 / \mathrm{cnt} / \mathrm{cnt} 4$.pdf.

BEYEBACH, M. (1999). "Introducción a la terapia breve centrada en soluciones", en G. Navarro y G. Fuentes (eds.), Intervención y prevención en salud mental. España: Amarú.

BuRín, M. (1999) "Género y psicoanálisis: subjetividades femeninas vulnerables", en M. Burin y E. Dio Bleichmar (comps.), Género, psicoanálisis, subjetividad, pp. 61-99. Buenos Aires: Paidós.

ButLER, J. (2006). Deshacer el género. Barcelona: Paidós.

CAPUTI, J. (1993). "American psychos: the serial killer in contemporary fiction", en Journal of American Culture, vol. 16, núm. 4, pp. 101-112.

CASTRO, R. y BRONFMAN, M. (1993). “Teoría feminista y sociología médica: bases para una discusión", en Cad. Saúde Públ., vol. 9, núm. 3, pp. 375-394. CASTRO, R. y CACIQUE, I. (2008). "Introducción". En Roberto Castro e Irene Cacique (edits.), en Estudios sobre cultura, género y violencia contra las mujeres, pp. 11-15. México: UNAM.

CASTILLO M., R. y MONTES B., B. (2007). “Validación de las escalas relacionadas con la socialización del género", en Iniciación a la Investigación, vol. 2, pp. 1-9.

CAZÉS, D. (2005). La misoginia: ideología de las relaciones humanas. Una introducción, en D. Cazés y F. Huerta (coords.), Hombres ante la misoginia: miradas críticas. México: Plaza y Valdés / CEIICH-UNAM. 


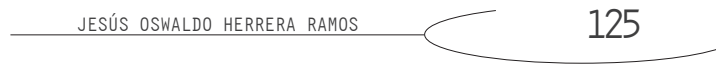

Código Penal Federal (2011). Diario Oficial de la Federación. Recuperado el 14 de mayo de 2011 de: http://www.diputados.gob.mx/LeyesBiblio/ pdf/9.pdf.

CORSI, J. (2003). "La violencia en el contexto familiar como problema social", en J. Corsi (comp.), Maltrato y abuso en el ámbito doméstico, pp. 15-40. Argentina: Paidós.

ECHEBURÚA, E. y FERnÁNDEZ-MONTALVO, J. (1998). "Hombres maltratadores", en E. Echeburúa y P. de Corral (coords.), Manual de violencia familiar. España: Siglo XXI.

FOUCAULT, M. (1977). Historia de la sexualidad 1: la voluntad de saber. México: Siglo XXI.

- (1980)."Two Lectures", en Power/Knowledge: Selected Interviews and Other Writings, pp. 78-108. Nueva York: Pantheon, .

Gargallo, F. (2006). Ideas feministas latinoamericanas. Venezuela: El Perro y la Rana.

Gómez-Jarabo, G., Rodríguez, E. y OlavarrietA, S. (2004). “Valoración de la capacidad de obrar: el control de los impulsos", en Investigación en Salud, vol. 6, núm. 1, pp. 35-42.

HARE, R. (2003). Sin conciencia. El inquietante mundo de los psicópatas que nos rodean. España: Paidós.

HerRero, O. y Colom, R. (2006). “¿Es verosímil la teoría de la delincuencia de David Lykken?", en Psichotema, vol. 18, núm. 3, pp. 374-377.

HIKAL, W. (2009). "Criminología del desarrollo: el estudio de la personalidad", en Ciencia UANL, vol. 12, núm. 2, pp. 124-130.

JÁUREGUI, I. (2008). "Psicopatía, ideología y sociedad", en Nómadas, Revista Crítica de Ciencias Sociales y Jurídicas, vol. 18. Recuperado el 18 de 
abril del 2011 de: http://redalyc.uaemex.mx/redalyc/pdf/181/18101805. pdf.

KAUFMAN, M. (1994) “Men, feminism, and men's contradictory experiences of power", en H. Brod y M. Kaufman (eds.), Theorizing Masculinities, pp. 142-163. California: Sage.

LAGARDE, M. (2008). "Antropología, feminismo y política: violencia feminicida y derechos humanos de las mujeres", en M. Bullen y C. Diez (coords.), Retos teóricos y nuevas prácticas. México: Ed. Ankulegi Antropología Elkartea. Recuperado el 22 de marzo del 2011 de: http://www.euskomedia.org/PDFAnlt/antropologia/11/14/14209239.pdf.

LAMAS, M. (2002). Cuerpo: diferencia sexual y género. México: Taurus.

- (2007)."Las putas honestas, ayer y hoy", en M. Lamas (comp.), Miradas feministas sobre las mexicanas del siglo XX, pp. 312-346. México: Fondo de Cultura Económica.

Ley General de ACCeso de las Mujeres A unA VidA LiBre de ViOlenCia (2011). Diario Oficial de la Federación. Recuperado el 17 de marzo del 2011 de: http://www.diputados.gob.mx/LeyesBiblio/pdf/LGAMVLV.pdf.

MedinA-MorA, M. et al. (2003). "Prevalencia de trastornos mentales y uso de servicios: resultados de la encuesta nacional de epidemiología psiquiátrica en México", en Salud Mental, vol. 26, núm. 4, pp. 1-16.

Millon, T., Grossman, S., Millon, C., Meagher, S. y Ramnath, R. (2004). Personality disorders in modern life. New Jersey: $2^{\mathrm{a}}$ ed., John Wiley \& Sons, Inc.

Minello, N. (2005). "De la misoginia y otras dominaciones", en D. Cazés y F. Huerta (coords.), Hombres ante la misoginia: miradas críticas, pp. 77-86. México: Plaza y Valdés / CEIICH-UNAM. 


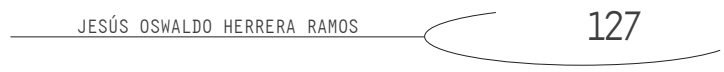

MolinA, J., TRABAZO, V., LÓPEZ, L. Y FernándeZ, S. (2009). “Delictología de los trastornos de personalidad y su repercusión sobre la imputabilidad", en EduPsykhé. Revista de Psicología y Educacion, vol. 8, núm. 2, pp. 101126.

ORDORIKA, T. (2009). "Aportaciones sociológicas al estudio de la salud mental de las mujeres", en Revista Mexicana de Sociología, vol. 71, núm. 4, pp. 647-674.

PERRONE, R. y NANNINI, M. (2007). Violencia y abusos sexuales en la familia. Argentina: $2^{\mathrm{a}}$ ed., Paidós.

PHILLIPS, K. y GUNDERSON, J. (1996). "Trastornos de la personalidad", en R. Hales, S. Yudofsky y J. Talbott (coords.), Tratado de Psiquiatría. Estados Unidos: $2^{\mathrm{a}}$ ed., American Psychiatric Press.

QUIROGA, E. y ERRASTI, J. (2001). “Tratamientos psicológicos eficaces para los trastornos de personalidad", en Psicothema, vol. 13, núm. 3, pp. 393-406.

RUSSELL, D. (2006) “Feminicidio: la 'solución final' de algunos hombres para las mujeres", en D. Russell y R. Harmes (eds.), Feminicidio: una perspectiva global. México: CEIICH-UNAM.

SAldívar, G., RAMOS, L. y SAltIJeral, M. (2004). "Validación de las escalas de violencia en universitarios", en Salud Mental, vol. 27, pp. 40-49.

SAUCEDO, I. (2003). "Violencia de género: problema prioritario de salud pública. Oportunidades y límites para la puesta en marcha de programas en el sistema de salud del Estado", en P. López, B. Rico, A. Langer y G. Espinoza (comps.), Género y política en salud, pp. 359-388. México: Secretaría de Salud. 
SCOTT, J. (1990). "El género: una categoría útil para el análisis histórico", en J. Amelang y M. Nash (eds.), Historia y género: las mujeres en la Europa Moderna y Contemporánea, pp. 23-56. España: Alfons el Magnànim.

SPITZER, R., GIBBON, M., SKODOL, A., WILLIAMS, J. y FIRST, M. (2003). DSM-IV-TR libro de casos: compañero del DSM-IV-TR. España: Masson.

SZASZ, T. (2002). Esquizofrenia. El símbolo sagrado de la psiquiatría. México: Ediciones Coyoacán.

TiRAdo-ÁlvareZ, M. (2010). “Necesidad de la creación de una sanción penal especial para ser impuesta al sujeto que padece trastorno antisocial de la personalidad (psicopatía) en Colombia", Revista Estudios Socio-Jurídicos, vol. 12, núm. 1, pp. 127-154.

VEGA, Z. (2004). "Las alteraciones o perturbaciones psíquicas como causas de inimputabilidad: especial problemática en el ámbito de las psicopatías", en Memorias del encuentro No. 70. Managua, Nicaragua: Universidad Centroamericana. Recuperado el 8 de junio del 2011 de: http://bibliotecavirtual.clacso.org.ar/ar/libros/nicaragua/uca/encuen70/vega.rtf. ZUMAYA, M. (2005). "Psiquiatras, psicoanalistas y otros misóginos", en D. Cazés y F. Huerta (coords.), Hombres ante la misoginia: miradas críticas. México: Plaza y Valdés / CEIICH-UNAM. 